\title{
OFDM Frequency Offset Estimation Based on BLUE Principle
}

\author{
H. Minn, Member, IEEE, P. Tarasak, Student Member, IEEE, \\ and V.K. Bhargava*, Fellow, IEEE \\ Department of Electrical and Computer Engineering \\ University of Victoria \\ Victoria, B.C., Canada V8W 3P6 \\ Tel: +1 2507216043 , Fax: +1 2507216048 , \\ Email: bhargava,hminn,ptarasak@ece.uvic.ca
}

\begin{abstract}
High performance frequency estimation is an important issue in orthogonal frequency division multiplexing (OFDM) systems since OFDM systems are very sensitive to carrier frequency offsets. Recently, Morelli \& Mengali (M\&M) [12] presented a near optimal frequency estimator based on the best linear unbiased estimation (BLUE) principle. In this paper, we have presented three frequency estimation methods based on the BLUE principle. The third method has the same frequency estimation mean square error (MSE) performance as the M\&M method. The first two methods give better MSE performance than the M\&M method especially at low SNR values. The first two methods' MSE performances are essentially the same and quite close to the Cramer-Rao lower bound for all SNR values, hence, these proposed methods are very attractive for OFDM applications.
\end{abstract}

Index Terms-OFDM, Frequency offset, Frequency synchronization, BLUE.

\section{INTRODUCTION}

Orthogonal frequency division multiplexing (OFDM) has achieved a wider acceptance in many applications such as Digital Audio Broadcasting (DAB) [1], Digital Video Broadcasting (DVB) [2], wireless local area network and short range wireless access standards (IEEE 802.11a [3], HiperLAN/2 [4], Multimedia Mobile Access Communication (MMAC) [5], and wireless metropolitan area network and broadband wireless access standard (IEEE 802.16a/b) [6]. One of the main drawbacks of OFDM is its high sensitivity to frequency offsets caused by the oscillator inaccuracies and the Doppler shift of the mobile channel [7]. As the frequency offset induced phase error accumulates over the successive symbols, the frequency offset estimation accuracy becomes more important for a system with a larger packet length.

Several schemes (e.g., [8] - [12]) have been proposed for OFDM frequency offset estimation. The cyclic prefix based approaches such as [9], [10] would be suitable for continuous transmission while training symbol based approaches such as [8] [11] and [12] could be used in either continuous transmission or packet oriented burst transmission. In [8], a maximum likelihood frequency offset estimator was presented based on the use of two consecutive and identical symbols. The maximum frequency offset that can be handled is $\pm 1 / 2$ of the subcarrier spacing. The

*Contact Author

This work was supported, in part, by a Strategic Project Grant from the Natural Sciences and Engineering Research Council (NSERC) of Canada. method of [11] also applied two training symbols. The first symbol has two identical halves and is used to estimate an frequency offset less than the subcarrier spacing. The second symbol contains a pseudonoise sequence and is used to resolve the ambiguity resulting from the estimation based on the first symbol. Recently in [12], Morelli and Mengali (M\&M) presented an improved frequency offset estimation based on the best linear unbiased estimation (BLUE) principle. The M\&M method uses a training symbol composed of $L>2$ identical parts and the frequency acquisition range is $\pm L / 2$ of the subcarrier spacing. Its frequency estimation MSE performance is quite close to the Cramer-Rao bound (CRB).

In this paper, we derive a different frequency offset estimator but use the same training symbol composed of $L$ identical parts and the BLUE principle as in the M\&M method. We present three methods for the frequency offset estimation. All proposed approaches have the same frequency acquisition range of $\pm L / 2$ the subcarrier spacing as the M\&M method. The first and the second methods have better MSE performance than the M\&M method. The third method has the same MSE performance as the M\&M method. The rest of this paper is organized as follows. Section II describes the signal model. Section III presents the proposed frequency offset estimation and the three methods to implement it. Simulation results are discussed in Section IV. Finally, conclusions are given in Section V.

\section{SignAl MODEL}

The time-domain complex baseband samples $\{s(k)\}$ of the useful part of an OFDM signal with $N$ subcarriers are generated by taking the $N$-point inverse fast Fourier transform $\left(\mathrm{IFFT}_{N}\right)$ of a block of subcarrier symbols $\left\{C_{l}\right\}$ which are from a QAM or PSK signal constellation as

$$
s(k)=\frac{1}{\sqrt{N}} \sum_{l=-N_{u}}^{N_{u}} C_{l} e^{j 2 \pi l k / N}, \quad 0 \leq k \leq N-1
$$

where the number of used subcarriers is $2 N_{u}+1 \leq N$.

The useful part of each OFDM symbol has a duration of $T$ seconds and is preceded by a cyclic prefix, which is longer than the channel impulse response, in order to avoid inter-symbol interference (ISI). Assuming that the timing synchronization eliminates 
the ISI, the receive filter output samples $\{r(k)\}$ taken at the sampling rate of $N / T$ can be given by

$$
r(k)=e^{j 2 \pi v k / N} x(k)+n(k)
$$

where $v$ is the carrier frequency offset normalized by the subcarrier spacing $1 / T, n(k)$ is a sample of complex Gaussian noise process with zero mean and variance $\sigma_{n}^{2}=E\left\{|n(k)|^{2}\right\}$ and $x(k)$ is the channel output signal component given by

$$
x(k)=\frac{1}{\sqrt{N}} \sum_{l=-N_{u}}^{N_{u}} C_{l} \Gamma_{l} e^{j 2 \pi l k / N}, 0 \leq k \leq N-1
$$

where $\Gamma_{l}$ is the total frequency response at the $l^{\text {th }}$ subcarrier, including the effects of the channel, filters, timing offset and arbitrary carrier phase factor. The signal-to-noise ratio is defined as $\mathrm{SNR} \triangleq \sigma_{x}^{2} / \sigma_{n}^{2}$, with $\sigma_{x}^{2} \triangleq E\left\{|x(k)|^{2}\right\}$.

The frequency offset estimation is based on the training symbol $\{s(k)\}$ consisting of $L$ identical parts. The training symbol can be generated by transmitting a pseudonoise sequence on the subcarriers whose indexes are multiples of $L$ and setting zero on the remaining subcarriers. In other words, the training symbol $\{s(k)\}$ is generated by $\operatorname{IFFT}_{N}$ of $\left\{C_{l}\right\}$ where $\left\{C_{l=i L}\right\}$ is of a pseudonoise sequence and $\left\{C_{l \neq i L}\right\}=0$.

\section{FREQuency OfFSET Estimation}

The proposed frequency offset estimation is based on the correlations among the identical parts of the received training symbol. Define the correlation term as

$$
R(m)=\sum_{k=0}^{N-m M-1} r^{*}(k) r(k+m M), \quad 1 \leq m \leq H
$$

where $M=N / L$ is the number of the samples of each identical part of the training symbol and $H$ is a design parameter with $1 \leq$ $H \leq L-1$. Substituting (2) into (4) results in

$$
R(m)=e^{j 2 \pi v m M / N}\left\{(L-m) E_{1}+G(m)+\mathcal{N}(m)\right\}
$$

where

$$
\begin{aligned}
E_{1} & \triangleq \sum_{k=0}^{M-1}|x(k)|^{2} \\
G(m) & \triangleq \sum_{k=0}^{N-m M-1}\left\{x^{*}(k) \tilde{n}(k+m M)+\right. \\
\mathcal{N}(m) & \left.\triangleq \sum_{k=0}^{N-m M-1} \tilde{n}^{*}(k) \tilde{n}(k+m M) \tilde{n}^{*}(k)\right\}
\end{aligned}
$$

and $\tilde{n}(k) \triangleq n(k) e^{-j 2 \pi v k / N}$ is a random variable statistically equivalent to $n(k)$.

Define the following:

$$
\theta_{m} \triangleq \frac{N}{2 \pi m M} \arg \{R(m)\} .
$$

If $|v|<N /(2 m M)$, then we have

$$
\theta_{m}=v+\frac{N}{2 \pi m M} \arg \left\{(L-m) E_{1}+G(m)+\mathcal{N}(m)\right\}
$$

and hence, $\theta_{m}$ gives an estimate of $v$. For smaller $m$ values, $M$ can be designed to handle the possible maximum frequency offset, i.e., to satisfy the condition $|v|<N /(2 m M)$. However, the oscillator inaccuracies and the channel Doppler shift may not guarantee the condition $|v|<N /(2 m M)$ for larger $m$ values. Hence, $\theta_{m}$ with larger $m$ values are associated with an ambiguity problem and would not be suitable for use as an estimate for $v$. To circumvent this, we propose the following. First, $\theta_{1}$ is calculated and used as an initial estimate of $v$. Then the initial frequency offset compensation is performed on the received training symbol by using the initial frequency offset estimate $\theta_{1}$. The frequency offset compensated received training symbol sample $\tilde{r}(k)$ can be expressed as

$$
\tilde{r}(k)=r(k) e^{-j 2 \pi \theta_{1} k / N} .
$$

Using $\tilde{r}(k)$ in place of $r(k)$ in (4) and (9) gives

$$
\begin{aligned}
& \tilde{R}(m)=\sum_{k=0}^{N-m M-1} \tilde{r}^{*}(k) \tilde{r}(k+m M), 2 \leq m \leq H \\
& =e^{j 2 \pi\left(v-\theta_{1}\right) m M / N}\left\{(L-m) E_{1}+\tilde{G}(m)+\tilde{\mathcal{N}}(m)\right\}
\end{aligned}
$$

and

$$
\begin{aligned}
\tilde{\theta}_{m}= & \frac{N}{2 \pi m M} \arg \{\tilde{R}(m)\}, 2 \leq m \leq H \\
= & \left(v-\theta_{1}\right)+\frac{N}{2 \pi m M} \arg \left\{(L-m) E_{1}\right. \\
& +\tilde{G}(m)+\tilde{\mathcal{N}}(m)\}
\end{aligned}
$$

where $\tilde{G}(m)$ and $\tilde{\mathcal{N}}(m)$ have the same statistical behaviors as $G(m)$ and $\mathcal{N}(m)$, respectively. Since $\theta_{1}$ would be close to $v$, $\left\{\tilde{\theta}_{m}: 2 \leq m \leq H\right\}$ give estimates of $\left(v-\theta_{1}\right)$ without any ambiguity. Now, $\left\{\theta_{m}: 2 \leq m \leq H\right\}$ can be given by

$$
\theta_{m}=\theta_{1}+\tilde{\theta}_{m}, \quad 2 \leq m \leq H .
$$

The frequency offset estimator based on the BLUE principle can then be given by [13]

$$
\hat{v}=\sum_{m=1}^{H} w_{m} \theta_{m}
$$

where $w_{m}$ is the $m^{t h}$ component of the weighting vector

$$
\boldsymbol{w}=\frac{C_{\theta}^{-1} \mathbf{1}}{\mathbf{1}^{T} C_{\theta}^{-1} \mathbf{1}}
$$


Here, $\boldsymbol{C}_{\theta}$ is the covariance matrix of $\boldsymbol{\theta} \triangleq\left[\theta_{1}, \theta_{2}, \ldots, \theta_{H}\right]^{T}$ and 1 is an all ones column vector of length $H$. The variance of the BLUE is given by [13]

$$
\operatorname{var}\{\hat{v}\}=\frac{1}{\mathbf{1}^{T} \boldsymbol{C}_{\theta}^{-1} \mathbf{1}} .
$$

The above frequency offset estimation based on the BLUE principle requires $\boldsymbol{C}_{\theta}$. In the following, three methods are presented for obtaining the required (approximate) value of $\boldsymbol{C}_{\theta}$.

\section{A. Method A}

The analytical calculation of $\boldsymbol{C}_{\theta}$ is quite intractable and hence, a simulation based approach is resorted in Method A. Since $v$ is the unknown parameter to be estimated, the $\boldsymbol{C}_{\theta}$ is evaluated by simulation with $v=0$. The channel is also unknown and hence, $\boldsymbol{C}_{\theta}$ is evaluated for additive white Gaussian noise (AWGN) channel. Note that we have also evaluated $\boldsymbol{C}_{\theta}$ with the known channel response and the results are essentially the same. Due to the uncorrelation of the Gaussian noise samples, it can readily be concluded that $\boldsymbol{C}_{\theta}$ is of full rank for $1 \leq H \leq L-1$. From (17), it can be readily found that the maximum value of $H$ for the full rank $\boldsymbol{C}_{\theta}$ will give the minimum variance. In this simulation-based approach, a design SNR value, $\mathrm{SNR}_{w}$, has to be used. However, the best $\mathrm{SNR}_{w}$ value is unclear at this point and will be investigated in the next section.

\section{B. Method B}

We can express $\theta_{m}$ as

$$
\theta_{m}=v+\frac{N}{2 \pi m M} \tan ^{-1}\left(\frac{G_{I}(m)+\mathcal{N}_{I}}{(L-m) E_{1}+G_{R}(m)+\mathcal{N}_{R}}\right)
$$

where $X_{R}$ and $X_{I}$ are, respectively, the real and the imaginary parts of $X$ and $\tan ^{-1}()$ is the four quadrant arctangent function.

For high SNR values, we have $(L-m) E_{1}+G_{R}(m)+\mathcal{N}_{R}$ $\simeq(L-m) E_{1}$ and $\tan ^{-1}\left(\frac{G_{I}(m)+\mathcal{N}_{I}}{(L-m) E_{1}}\right) \simeq \frac{G_{I}(m)+\mathcal{N}_{I}}{(L-m) E_{1}}$. Then $\theta_{m}$ can be approximately expressed as

$$
\theta_{m} \simeq v+\frac{N}{2 \pi m M} \frac{G_{I}(m)+\mathcal{N}_{I}}{(L-m) E_{1}} .
$$

By using (19), the $m^{\text {th }}$ row, $n^{\text {th }}$ column element of $\boldsymbol{C}_{\theta}$ can be calculated as (20) shown at the bottom of this page.

It can be readily checked that $C_{\theta}$ is of full rank for $1 \leq$ $H \leq L-1$. By substituting (20) into (17), it can be found that $H=L-1$ gives the minimum variance. In practice, the value of SNR required in (20) can be replaced by the designed SNR value $\mathrm{SNR}_{w}$. The effect of mismatch between SNR and $\mathrm{SNR}_{w}$ will be investigated in the next section.

\section{Method C}

Similar to Method B, another high SNR approximation of $\theta_{m}$ can be given by

$$
\theta_{m} \simeq v+\frac{N}{2 \pi m M} \frac{G_{I}(m)}{(L-m) E_{1}} .
$$

The corresponding elements of $\boldsymbol{C}_{\theta}$ can be calculated as

$$
\begin{aligned}
\boldsymbol{C}_{\theta}(m, n) & =\frac{N^{2}}{4 \pi^{2} M^{3}} \frac{1}{S N R} \frac{1}{m n(L-m)(L-n)} \\
& \times \begin{cases}\min (m, n) & \text { if } m+n<L \\
L-\max (m, n) & \text { if } m+n \geq L\end{cases}
\end{aligned}
$$

From (22), it can be observed that $\boldsymbol{C}_{\theta}$ has mirror properties about $(L / 2)^{t h}$ row and $(L / 2)^{t h}$ column, i.e., $(L / 2+k)^{t h}$ row/column is the same as $(L / 2-k)^{t h}$ row/column. Hence, $\boldsymbol{C}_{\theta}$ is of full rank only for $1 \leq H \leq L / 2$. It can also be checked that $H=L / 2$ gives the minimum variance. For this method, SNR value is not required in the calculation of $\boldsymbol{w}$, contrary to Method $\mathrm{A}$ and $\mathrm{B}$.

\section{Simulation Results and Discussions}

Simulations have been carried out to evaluate the estimation performance of the proposed methods. The simulation parameters are the same as those in [12]: $N=1024,2 N_{u}+1=861$, a cyclic prefix of 40 samples and a multipath Rayleigh fading channel. The channel has 25 paths with the path delays of $0,1, \ldots, 24$ samples and an exponential power delay profile with the power of $i^{t h}$ path equal to $\exp (-i / 5)$. The number of identical parts of the training symbol is $L=8$ and the normalized frequency offset is set to $v=1.6$.

In Fig. 1, the variances of the BLUE for the three methods are plotted for all values of $H$. Since Method B and C use some approximation in obtaining the covariance matrix, their variances just represent approximate values. From Fig. 1, we can observe that the maximum value of $H$ gives the minimum variance for all methods, i.e., $H=7$ for Method A and B and $H=4$ for Method C.

In Fig. 2(a) and (b), the weighting values $\left\{w_{m}\right\}$ for the number of weighting taps $H=4$ and $H=7$, respectively, are presented. It can be observed from Fig. 2(a) that the tap weights of Method B for $H=L / 2=4$ change considerably as the values of $\mathrm{SNR}_{w}$ change. For $H=L-1=7$, as can be seen from Fig. 2(b), the tap weights of both Method A and B are virtually not affected by the values of $\mathrm{SNR}_{w}$. The weight values of Method A for $H=L-1$ are virtually the same as those of Method B. Regarding the weighting values, some discussion follows. From (10), it can be observed that $\theta_{m}$ with a larger value of $m$ has more reliability, if the number of correlation samples are the same, due to the factor $\frac{N}{2 \pi m M}$. However, $\theta_{m}$ with a larger value of $m$ has a

$$
\boldsymbol{C}_{\theta}(m, n)=\frac{N^{2}}{4 \pi^{2} M^{3}} \frac{1}{S N R} \frac{1}{m n(L-m)(L-n)} \times \begin{cases}m+\frac{L-m}{2 S N R} & \text { if } m=n \& m<L / 2 \\ (L-m)+\frac{L-m}{2 S N R} & \text { if } m=n \& m \geq L / 2 \\ \min (m, n) & \text { if } m \neq n \& m+n<L \\ L-\max (m, n) & \text { if } m \neq n \& m+n \geq L\end{cases}
$$


(a) Method A

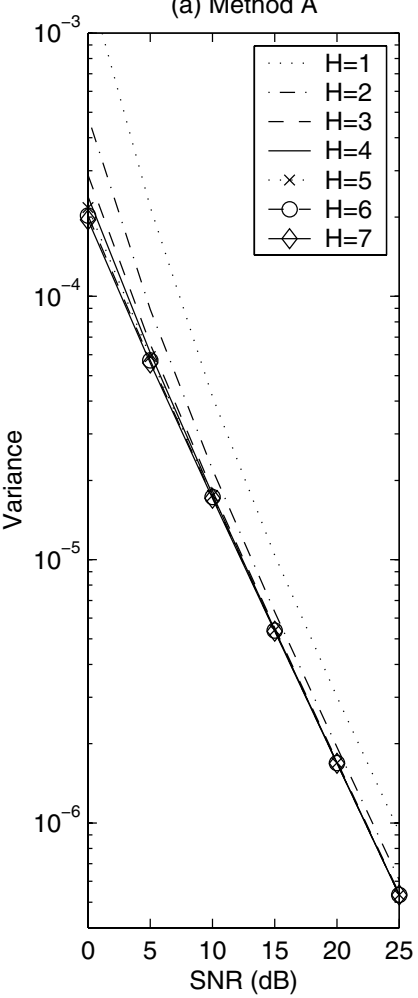

(b) Method B

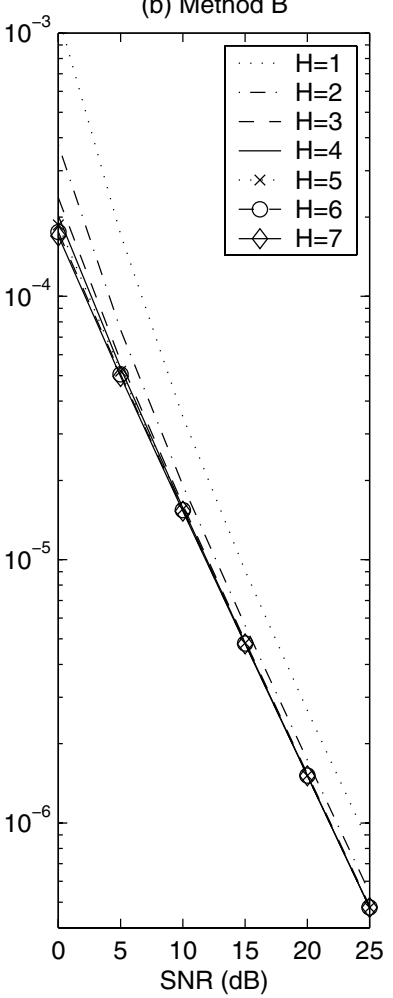

(c) Method C

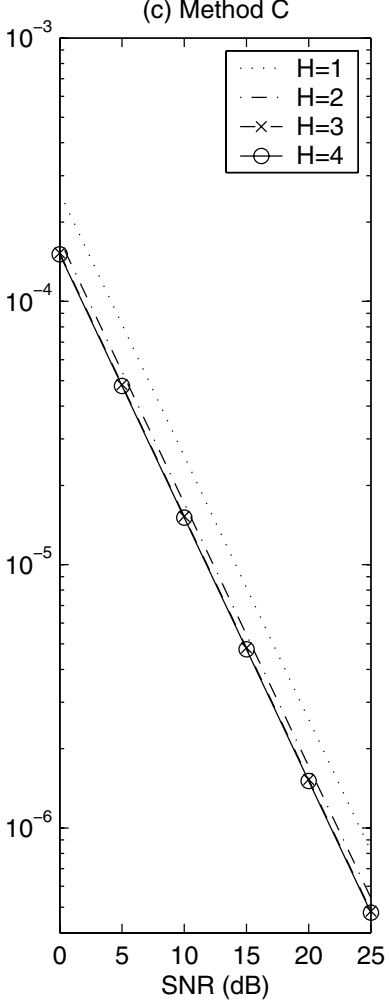

Fig. 1. The variances of the BLUE for the three methods

smaller number of correlation samples, $N-m M$, which indicates less reliability. These two conflicting effects shape the weighting values which can be viewed as the reliabilities of $\left\{\theta_{m}\right\}$.

Fig. 3 shows the simulation results for the frequency offset estimation MSE performance of the three proposed methods and the M\&M method. For the M\&M method, $H=L / 2$, which gives the minimum variance, is used. The MSE performance of Method $\mathrm{C}$ is the same as that of M\&M method. Although the estimators are different, both use the BLUE principle and the same approximation in calculating the weighting values, resulting in the same result. For high SNR values, all methods have virtually the same MSE performance. As the SNR value becomes smaller, Method B with $H=4$ achieves slightly better MSE performance than the M\&M method, and both Method A with $H=7$ and Method B with $H=7$ achieve considerably better MSE performance than the M\&M method. Method A and B with $H=7$ have essentially the same MSE performance since their weighting values are essentially the same. Also included for comparison in the figure is the CRB given by [12]

$$
C R B(\hat{v})=\frac{1}{2 \pi^{2} S N R} \frac{3}{N\left(1-1 / N^{2}\right)} .
$$

The M\&M method's MSE performance is quite close to the CRB especially for high SNR values. The proposed Method A and B with $H=7$ have the MSE performance quite close to CRB for all SNR values.

The MSE performances of Method A and B in Fig. 3 are obtained with $\mathrm{SNR}=\mathrm{SNR}_{w}$. However, in practice, there can be a mismatch between SNR and $\mathrm{SNR}_{w}$. Fig. 4 shows the MSE performance of Method B with mismatched $\mathrm{SNR}_{w}$ (i.e., $\mathrm{SNR}_{w} \neq \mathrm{SNR}$ ). Although the mismatched $\mathrm{SNR}_{w}$ can cause a very slight, trivial degradation for $H=4$, it essentially does not cause any MSE performance difference for $H=7$. This can also be observed from the fact that different $\mathrm{SNR}_{w}$ values give different weighting vector $\{\boldsymbol{w}\}$ for $H=4$ but virtually the same $\boldsymbol{w}$ for $H=7$. The mismatched $\mathrm{SNR}_{w}$ neither affects the frequency estimation performance of Method A with $H=7$ for the same reason and the corresponding simulation results are not included. Method A and $\mathrm{B}$ achieve better performance than Method $\mathrm{C}$ at the expense of some complexity.

\section{CONCLUSIONS}

Three frequency offset estimation methods based on the BLUE principle have been presented. In realizing the BLUE principle, the required covariance matrix is evaluated by simulation in the first method and approximated by high SNR assumption in the second and third methods. The first and second methods achieve better frequency estimation MSE performance than the near optimal estimator of Morelli \& Mengali (M\&M) [12]. The third method has the same MSE performance as the M\&M method. The first and the second methods have the same MSE performance and hence, the second method is more appealing since no simulation has to be performed in obtaining the weighting values. The proposed methods can be used not only in OFDM systems but also in single carrier systems. 

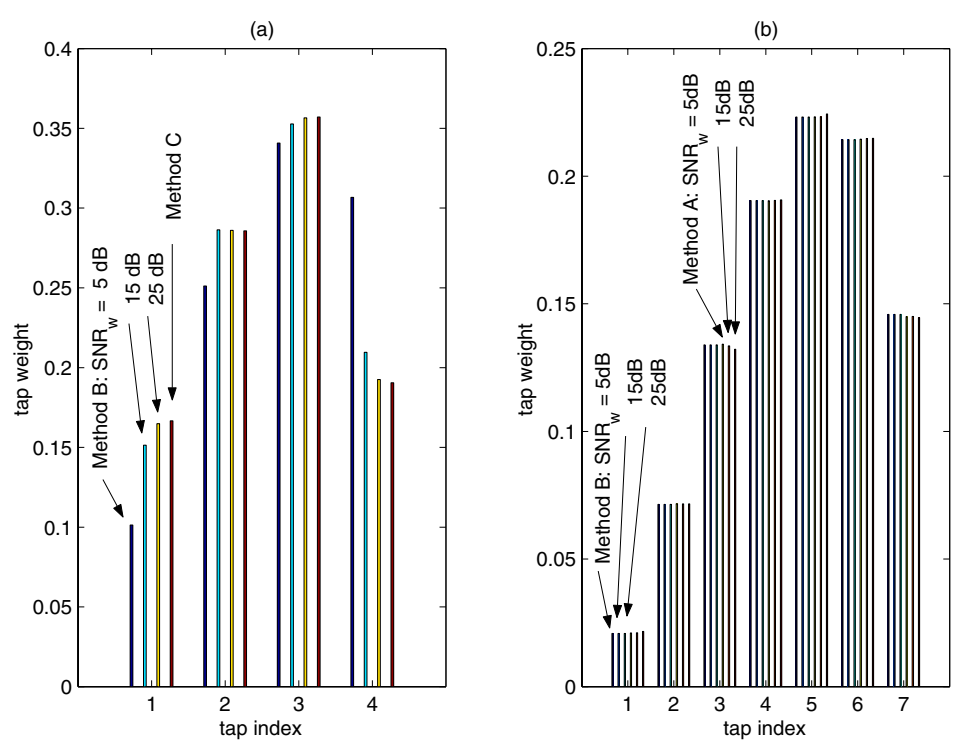

Fig. 2. The weighting values $\left\{w_{m}\right\}$ : (a) The number of weighting taps $H=4$ for Method B and C, (b) $H=7$ for Method A and B.

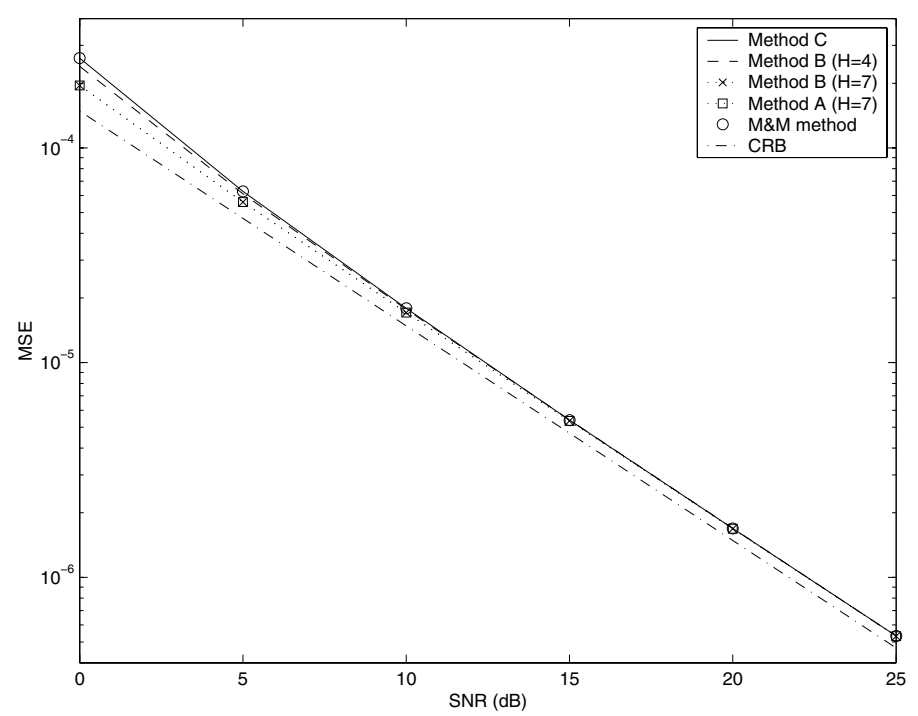

Fig. 3. The frequency estimation MSE performance comparison
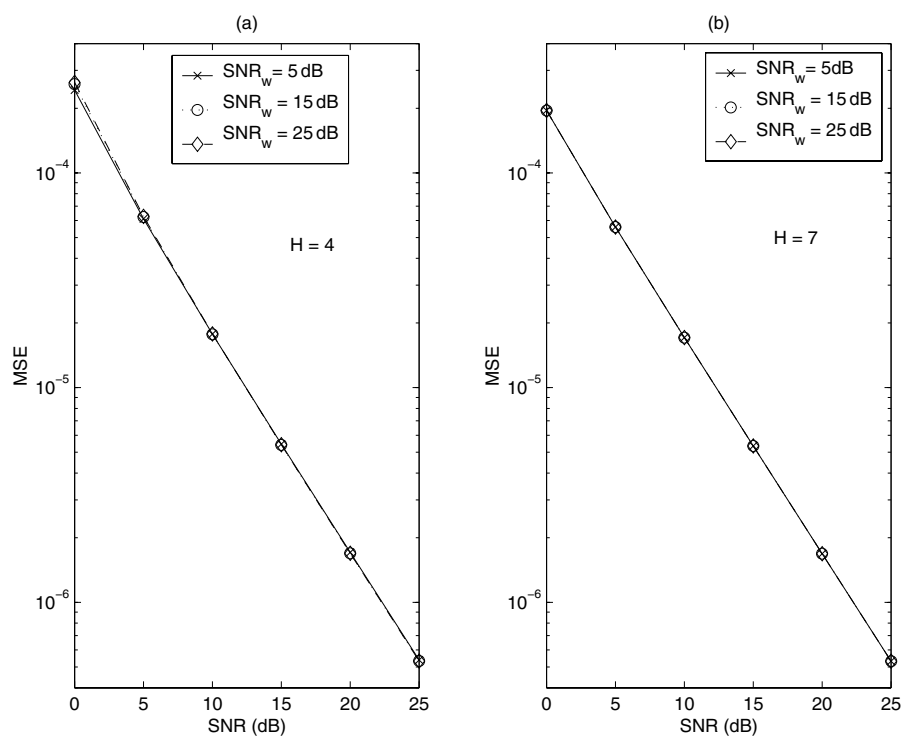

Fig. 4. The frequency estimation MSE performance of Method B with mismatched $\mathrm{SNR}_{w}$ (i.e., $\mathrm{SNR}_{w} \neq \mathrm{SNR}$ )

\section{REFERENCES}

[1] H. Sari, G. Karam and I. Jeanclaude, "Transmission techniques for digital terrestrial TV broadcasting," IEEE Commun. Mag., Vol. 33, Feb. 1995, pp. 100-109.

[2] ETSI, "Digital Video Broadcasting (DVB): Framing, channel coding and modulation for digital terrestrial television," ETSI EN300 744 V1.3.1 Draft (2000-08), 2000.

[3] IEEE LAN/MAN Standards Committee, "Wireless LAN medium access control (MAC) and physical layer (PHY) specifications: High-speed physical layer in the $5 \mathrm{GHz}$ band," IEEE Standard 802.11a, 1999.

[4] ETSI, "Broadband radio access networks (BRAN); HIPERLAN type 2; physical (PHY) layer technical specification," ETSI TS 101475 V1.1.1 (2000-04), 2000.

[5] "Multimedia Mobile Access Communication (MMAC)," http: www.arib.or.jp/mmac/e/index.htm.

[6] IEEE LAN/MAN Standards Committee, "IEEE 802.16 a/b (draft)," Broadband Wireless Access: IEEE MAN Standard, 2001.

[7] T. Pollet, M. Van Bladel and M. Moeneclaey, "BER sensitivity of OFDM systems to carrier frequency offset and Wiener phase noise," IEEE Trans. Commun., Vol. 43, No. 2/3/4, Feb./Mar./Apr. 1995, pp. 191-193.

[8] P.H. Moose, "A technique for orthogonal frequency division multiplexing frequency offset correction," IEEE Trans. Commun., Vol. 42, No. 10, Oct. 1994, pp. 2908-2914.

[9] F. Daffara and O. Adami, "A new frequency detector for orthogonal multicarrier transmission techniques," Proc. Vehicular Tech. Conf., Chicago, Illinois, USA, Jul. 1995, pp. 804-809.

[10] J-J. van de Beek, M. Sandell and P.O. Börjesson, "ML estimation of time and frequency offset in OFDM systems" IEEE Trans. Signal Proc., Vol. 45, no. 7, July 1997, pp. 1800-1805

[11] T. M. Schmidl and D. C. Cox, "Robust frequency and timing synchronization for OFDM," IEEE Trans. Commun., Vol. 45, No. 12, Dec. 1997, pp. 16131621.

[12] M. Morelli and U. Mengali, "An improved frequency offset estimator for OFDM applications," IEEE Commun. Letters, Vol. 3, No. 3, Mar. 1999, pp. 75-77.

[13] S. M. Kay, "Fundamentals of Statistical Signal Processing: Estimation Theory," Prentice Hall PTR, 1993. 